OPEN ACCESS

Edited by:

Xi-Nian Zuo,

Institute of Psychology (CAS), China

Reviewed by:

Yi Zeng,

Institute of Automation (CAS), China

Suiping Wang,

South China Normal University, China Domenica Romagno,

University of Pisa, Italy

${ }^{*}$ Correspondence:

Yanchao $B$

ybi@bnu.edu.cn

Specialty section:

This article was submitted to

Cognitive Science,

a section of the journal

Frontiers in Psychology

Received: 17 June 2017

Accepted: 24 August 2017

Published: 12 September 2017

Citation:

$X u$ Y, He Y and Bi Y (2017) A

Tri-network Model of Human Semantic

Processing. Front. Psychol. 8:1538.

doi: 10.3389/fpsyg.2017.01538

\section{A Tri-network Model of Human Semantic Processing}

\author{
Yangwen $\mathrm{Xu}$, Yong $\mathrm{He}$ and Yanchao $\mathrm{Bi}$ * \\ National Key Laboratory of Cognitive Neuroscience and Learning \& IDG/McGovern Institute for Brain Research, Beijing \\ Normal University, Beijing, China
}

Humans process the meaning of the world via both verbal and nonverbal modalities. It has been established that widely distributed cortical regions are involved in semantic processing, yet the global wiring pattern of this brain system has not been considered in the current neurocognitive semantic models. We review evidence from the brain-network perspective, which shows that the semantic system is topologically segregated into three brain modules. Revisiting previous region-based evidence in light of these new network findings, we postulate that these three modules support multimodal experiential representation, language-supported representation, and semantic control. A tri-network neurocognitive model of semantic processing is proposed, which generates new hypotheses regarding the network basis of different types of semantic processes.

Keywords: dual coding, module, hub, embodiment, language, control

\section{TOWARD A NETWORK PERSPECTIVE OF SEMANTIC PROCESSING}

Semantic memory contains general knowledge about the world, including objects, people, facts, and beliefs, that is abstracted away from specific experiences (Yee et al., 2013) and is crucial to a wide range of human cognitive functions including language, memory, object recognition and use, and reasoning. Semantic knowledge can be obtained and stored in various ways. Consider the concept of "Beijing." Someone who has never been in or seen anything about Beijing can deduce from linguistic contexts such as, "Beijing is the capital of China" that it is an important city that belongs to China. One may also know about Beijing by actually being there and experiencing it. These approaches of gaining knowledge about Beijing roughly correspond to two types of proposals about how semantic memory is developed and organized: one is based on experiences of various specific attributes, and the other is based on rich information supported by language, such as, word associations, word orders, and syntactic structures.

For the brain basis of semantic processing, decades of neuroimaging studies have consistently localized it to widely distributed brain regions across temporal, frontal, and parietal cortices (Binder et al., 2009). The conventional approach used by these studies is to identify regions activated by semantic tasks or lesion patterns associated with semantic deficits and to understand the function of each region in isolation. The prevailing models are dominated by the experience/attributebased representation of semantics, interpreting the regions that loosely belong to the sensorimotor cortices as representing semantic attributes of corresponding modalities (e.g., form, color, motion, sound, action, and emotion; Martin, 2016). Language is often considered as a processing modality in parallel to these modalities rather than as a system that makes special contributions to semantic representation (Patterson et al., 2007; Lambon Ralph et al., 2016). Regions outside the modalityspecific cortices or with functions related to semantic processing across modalities are often 
assumed to bind multiple attribute/modality-specific representations, e.g., the anterior temporal lobe (Patterson et al., 2007; Lambon Ralph et al., 2016) or the high-level convergence zones in the left temporal and inferior parietal regions (Binder and Desai, 2011), or to implement control processes that retrieve and manipulate semantic knowledge in a task- and context-appropriate fashion, e.g., the left frontoparietal and the left posterior temporal cortical areas (Jefferies, 2013; Lambon Ralph et al., 2016) or only the frontal regions (Binder and Desai, 2011). The functional assignments of specific roles to these regions are controversial and vary across models (Patterson et al., 2007; Binder and Desai, 2011; Jefferies, 2013; Lambon Ralph et al., 2016; Martin, 2016).

One important type of empirical evidence that was missing from the construction of a full neural model of semantic processing is the overall wiring structure, i.e., how the widely distributed semantic-related brain regions are topologically connected to support this complex faculty. Empirical profiling of the wiring pattern of the semantic system would not only provide direct evidence for how such diverse and distributed brain regions are communicated and incorporated but also pose important constraint on the understanding of the functions of individual regions, given that the functionality of a brain region is tightly related to its functional/structural connectivity patterns (Passingham et al., 2002). The importance of connectivity patterns has also been highlighted by previous models (Lambon Ralph et al., 2016; Martin, 2016), but only vague predictions about the global connectivity pattern could be derived: the modality/attribute-specific representations for a given concept are directly linked (Martin, 2016) or merged into higher-order representations in a graded manner (Lambon Ralph et al., 2016). The empirical evidence of the global wiring structure was absent, however, until recently.

The development of brain network analyses, advanced by the growing availability of techniques to measure brain connectivity and graph-theoretic approaches, offers a novel and global perspective to depict the topological organization of brain networks (Bullmore and Sporns, 2009; He and Evans, 2010; Sporns, 2013). A set of recent studies began to investigate the manner in which the widely distributed semantically relevant brain regions are connected, providing compelling clues about the organizational structure of the semantic system from a network viewpoint. This review seeks to highlight the recent empirical evidence about the network structure of the semantic system and to consider previous region-based studies in this new framework, leading to the proposal of a tri-network neurocognitive model of semantic processing. We will finally discuss how this network-based model generates new types of research avenues to study the neural basis of semantic processing.

\section{SEMANTIC FUNCTIONAL NETWORK: MODULES AND HUBS}

\section{Brain Networks and Graph Theory}

The global topological structure of a complex system can be quantified using various graph-theoretic measurements. Under this framework, the brain can be modeled as a network incorporating nodes and edges. The nodes correspond to brain regions that can be defined as regions of interest, e.g., building spheres around peaks obtained through activation studies (Power et al., 2011, 2013; Vandenberghe et al., 2013; Xu et al., 2016) or according to anatomical landmarks (Salvador et al., 2005; He et al., 2009; Fang et al., 2015). The edges correspond to interregional connections that can be measured by multiple non-invasive imaging techniques, such as, diffusion tensor imaging tracking white matter tracts (Basser et al., 1994), or the resting-state functional connectivity reflecting intrinsic functional coupling (Biswal et al., 1995).

By applying graph theory, the topological properties of the brain network can be measured quantitatively (Bullmore and Sporns, 2009; He and Evans, 2010). Two important network structures are modules and hubs (Figure 1). Modules refer to a community of nodes with internal connections that are much denser than those between communities. Various algorithms can be used to detect a modular structure in a network (Fortunato, 2010; Sporns and Betzel, 2016), e.g., hierarchical clustering or information-based theory. Hubs refer to nodes that have central roles in network communication, commonly identified as nodes with densest connections (van den Heuvel and Sporns, 2013). The role of hubs can be also described in terms of their connectivity arrangements in a modular structure (Guimera and Amaral, 2005; He et al., 2009; Power et al., 2013); provincial hubs have connections primarily to the nodes of their own module, while connector hubs have relatively even connections to the nodes of the modules they connect.

Modular and hub structure can provide important clues about the functional segregation and integration among brain regions (Sporns, 2013). Modularity analyses have revealed

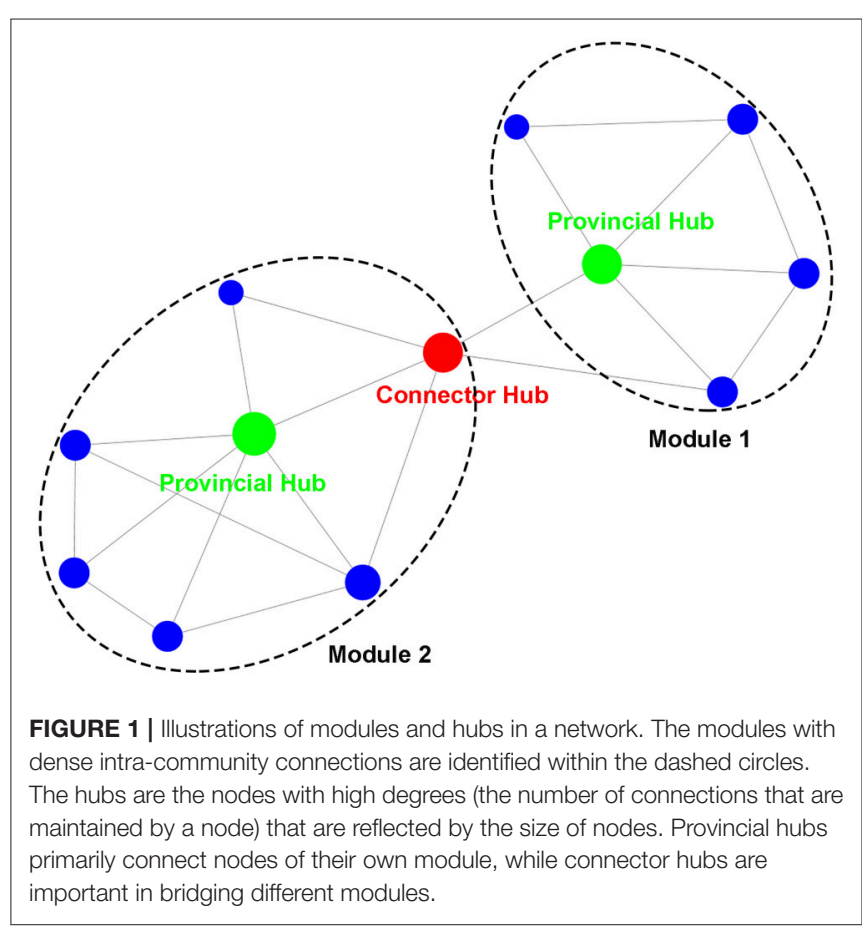


that the whole-brain intrinsic functional network can be consistently partitioned into segregated modules that correspond to dissociable cognitive components in the human mind, e.g., visual, somatomotor, default mode, dorsal/ventral attention, and control (He et al., 2009; Power et al., 2011; Yeo et al., 2011). Connector hubs linking different modules have been found to be essential to the integration of multiple cognitive functions, as damage to these regions was found to cause severe and widespread cognitive deficits (Warren et al., 2014).

\section{Brain Networks and the Semantic System}

The global topological structure of the semantic system was recently investigated. One study (Xu et al., 2016) constructed the intrinsic functional semantic network, with nodes defined as regions obtained from a careful and comprehensive meta-analysis (Binder et al., 2009) and edges defined as the inter-regional resting-state functional connectivity (Figure 2A, left). The meta-analysis results that were used to define nodes were based on 120 task-evoked neuroimaging studies that contain 187 semantic contrasts with orthographic and phonological processing demands and task difficulty matched for. The graph-theoretic approach was applied on this network, revealing three segregated modules (Figure 2A, middle), which were highly stable across datasets and various network construction methods (e.g., different nodal resolution-voxels or regions). According to the anatomical layout, these three modules were labeled as the left perisylvian network (PSN), the default mode network (DMN), and the left frontoparietal network (lFPN). Connector hubs that integrate different modules were also identified, e.g., the ATL was found to be the connector hub linking Modules DMN and PSN, while the posterior middle temporal gyrus (pMTG) was identified as the connector hub
A Network Connectivity

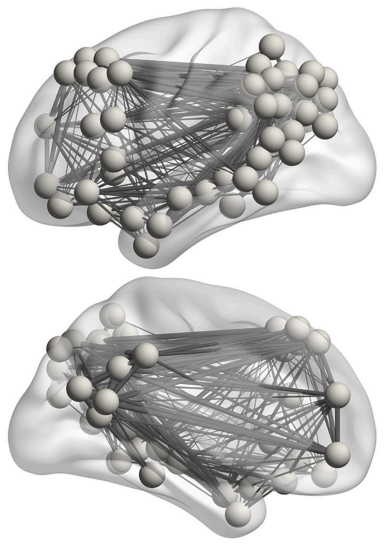

B (Areas Associated with 5 Semantic Attributes)

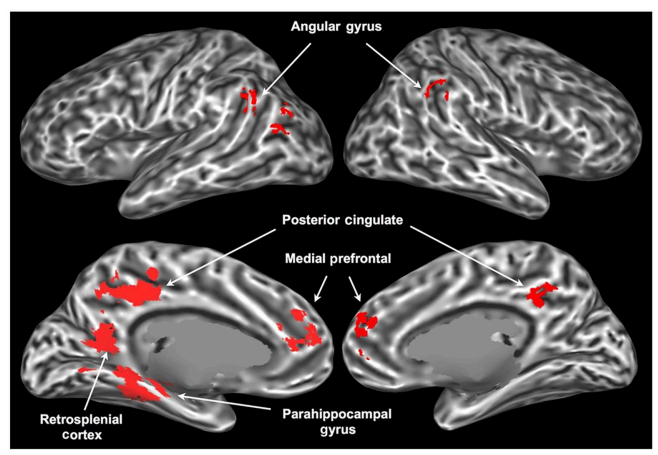

Network Modules

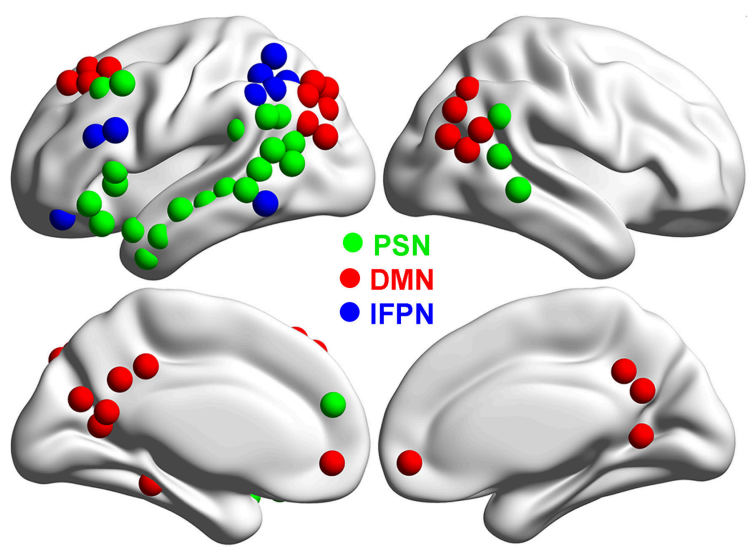

High-level Linguistic Processing (Sentence > Nonwords)

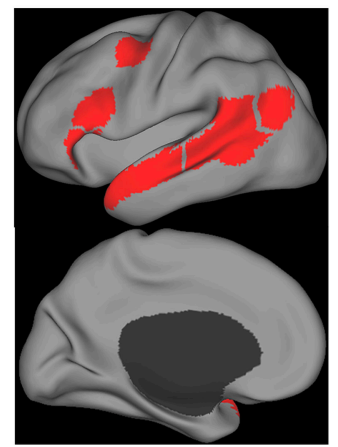

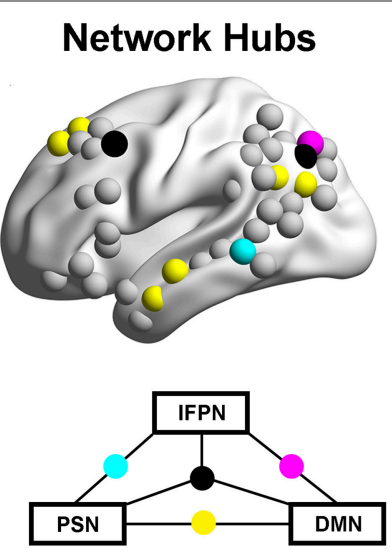

Semantic Control (High > Low Semantic Control)

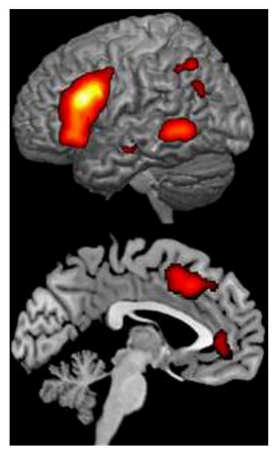

FIGURE 2 | Semantic functional network: modules, hubs, and their cognitive functions. (A) The organization of the intrinsic functional network of semantic processing Left: the semantic network showing nodes and edges, with nodes defined as the regions consistently activated during semantic processing obtained from a meta-analysis (Binder et al., 2009), and edges defined as the resting-state functional connectivity strength; Middle: the modules of the semantic network obtained by applying a graph-theoretic approach to the underlying connection patterns. Right: The connector hubs linking the three modules. Reproduced with permission from Xu et al. (2016). (B) Example results from task-evoked fMRI studies that shed light on the functions of the three modules. Left: The conjunction areas of five semantic aspects including shape, sound, motion, color, and manipulation from 900 words, which resemble the areas of Module DMN. Reprinted with permission from Fernandino et al. (2016); Middle: High-level linguistic processing regions generated from the group-level language localizer from 220 participants, which resemble the brain areas of Module PSN (https://evlab.mit.edu/funcloc/download-parcels); Right: The semantic control areas generated from a meta-analysis of 53 studies, which resemble the areas of Module IFPN. Reproduced with permission from Noonan et al. (2013). 
linking Modules DMN and FPN (Figure 2A, right). A similar approach was employed in a study (Fang et al., 2015) that constructed the structural semantic network by correlating the integrity of white matter tracts with the semantic performances in patients with brain damage. Although it was acknowledged that this constructed semantic structural network may not be complete owing to restricted lesion distributions (lack of posterior lesions), three modules were obtained in this study that aligned with those found in the intrinsic functional network (Xu et al., 2016): the "medial temporal lobe module" in the structural network functionally coincided with the Module DMN; the "orbital frontal-temporal/occipital module" overlapped with the Module PSN; the "opercular/triangular/middle frontalsubcortical module" corresponded to the Module FPN. Note that there was only one study that investigated the semantictask effects on the connectivity patterns (Vandenberghe et al., 2013). They first identified regions that were activated during an associative semantic task (the Pyramids and Palm Trees test). They then examined the functional connectivity pattern among these regions during the semantic and visuoperceptual control conditions. Six modules were detected, including one anatomically corresponding to the classical perisylvian language system, one to the visual perception system, and the other four that were difficult to label. Given that the functional connectivity was established based on both semantic and perceptual blocks, it was difficult to conclude whether the network structures were related to semantic or perceptual processing or both. Indeed, the visual module might be due to the visual tasks being employed and the observed perisylvian module converged onto the PSN module within the semantic system identified during the resting state (Xu et al., 2016).

Although there are only these few studies that directly addressed the global topological structure of the semantic system, clues about the semantic network structure could be gleaned from several other lines of researches. First, several recent studies focused on the connectivity pattern of specific semanticallyrelated regions as seeds, such as, the posterior and the middle part of the MTG (Turken and Dronkers, 2011; Wei et al., 2012; Davey et al., 2015, 2016; Feng et al., 2015), the ATL (Turken and Dronkers, 2011; Binney et al., 2012; Pascual et al., 2013; Feng et al., 2015; Jackson et al., 2016), the angular gyrus (Davey et al., 2015), the orbital and triangular part of the inferior frontal gyrus (IFG) (Saur et al., 2008; Turken and Dronkers, 2011; Feng et al., 2015), and the fusiform gyrus (Saur et al., 2008). They found that these regions have rich functional or structural connections with each other, and provide fragmented yet illuminating views about the overall patterns of the whole system, which converge with the network-level findings above. For example, consistent with the findings that the PMTG was the connector-hub between Modules PSN and FPN, seed-based studies showed that the pMTG has functional/structural connections with the brain areas in Modules PSN and Module FPN like the lateral temporal cortex, the IFG, the intraparietal sulcus (Turken and Dronkers, 2011; Wei et al., 2012; Davey et al., 2015, 2016; Feng et al., 2015). Also the seed-based studies found that the lateral ATL was functionally/structurally connected with the brain areas within the Modules DMN and PSN, such as, the ventral and anterior part of the IFG, the AG and the precuneus (Binney et al., 2012; Pascual et al., 2013; Feng et al., 2015; Jackson et al., 2016), consistent with the topological findings that the lateral ATL was the connector-hub between Modules PSN and DMN. Second, patient studies have focused on specific white-matter connections and revealed that disruptions in several large white-matter tracts, including the inferior fronto-occipital fasciculus, the anterior thalamic radiation, and the uncinate fasciculus that connect left temporal, frontal, parietal, and subcortical regions, are associated with semantic deficits (Duffau et al., 2005; Agosta et al., 2010; Acosta-Cabronero et al., 2011; Han et al., 2013). These results are in line with the rich intrinsic functional connections illustrated in Figure 2A (left). Finally, results about the whole-brain global network structure also tend to be in accord with the results focused only on semantic regions (He et al., 2009; Power et al., 2011; Yeo et al., 2011).

Intriguingly, the tri-module network structure is not naturally predicted or accounted for by any of the existing models of semantic processing, as outlined above. This network structure suggests the need to consider the functions of the semantic-related regions in the framework of the three modules-whether regions belonging to the same module have homogenous functions and what those functions might be. Notably, the existence of brain modular structure does not directly imply cognitive dissociation or synthesis-this is the classical reverse inference fallacy. Nonetheless, the cognitive hypotheses of semantic processing provide natural clues for interpreting the function of the brain network structure and together help formulate comprehensive neurocognitive models (Henson, 2005; Price and Friston, 2005; Poldrack, 2006). In the following section, we will review task-evoked neuroimaging and neuropsychological evidence about the functions of the brain regions in these three modules (summarized in Table 1) and postulate the functions of each module accordingly based on a broad range of pathological and functional neuroimaging data and existing meta-analyses results.

\section{SEGREGATED BRAIN MODULES, SEGREGATED SEMANTIC COMPONENTS}

\section{The DMN: The Multimodal Experiential System}

This module (red nodes in Figure 2A, middle) encompasses bilateral retrosplenial gyri/precuneus, bilateral medial prefrontal cortices, bilateral posterior angular gyrus (AG) extending to the superior division of the lateral occipital cortex, the left superior frontal gyrus (SFG), and the middle part of the left fusiform cortex/parahippocampal gyrus. These are the core regions of the DMN, originally defined as a brain system showing task-induced deactivation (Raichle et al., 2001).

The striking resemblance between the DMN and the semantic processing regions has long been noticed (Binder et al., 1999, 2009; Binder, 2012; Wei et al., 2012). Compared to the resting state, the DMN is significantly less deactivated for semantic tasks compared to perceptual or phonological tasks (Binder et al., 1999, 2009; Seghier et al., 2010; Wirth et al., 2011; Humphreys 


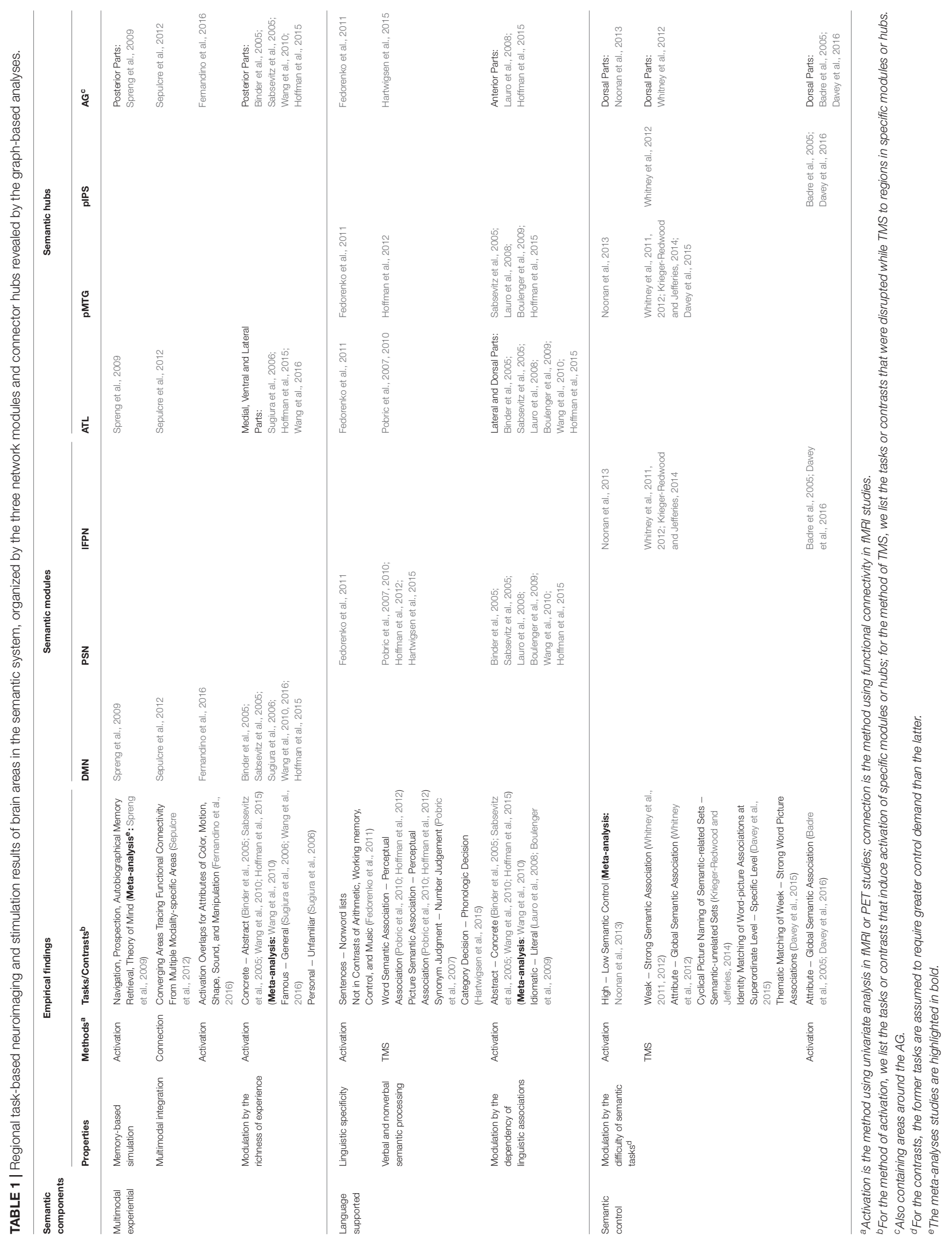


et al., 2015). Why is semantic processing special? One view is that the DMN does not process semantics and its activation during semantic processing is only the epiphenomenon of lower attention demand of semantic tasks relative to other tasks (Humphreys et al., 2015). In line with this view, the DMN plays a general intrinsic role, serving to maintain a functional balance with brain systems engaged in attention and control (Raichle, 2015). The magnitude of the DMN deactivation in visual tasks is related to the degree of task demand (Singh and Fawcett, 2008), and functional spontaneous fluctuations of the DMN was anticorrelated with the top-down attention network (Fox et al., 2005; Chai et al., 2012). Given that the DMN activation in semantic tasks was observed even when the task demand (defined by reaction time) of semantic processing is matched to (Wirth et al., 2011) or even stronger than (Seghier et al., 2010) the control tasks or when the task demand was explicitly regressed out (Binder et al., 2005), we suspect that the engagement of the DMN in semantic processing is not only due to the effects of general difficulty.

Another view is that DMN functionality is related to semantics. It has been considered that the resting state is not a blank state but rather involves "spontaneous cognitions," such as, remembering the past and thinking about the future, in which the DMN is recruited (Andrews-Hanna et al., 2010). Metaanalyses reveal that the DMN is the shared neural foundation of a spectrum of cognitive tasks, e.g., autobiographical memory retrieval, prospection, theory of mind, and navigation (Spreng et al., 2009). Considering the common cognitive component of these tasks, the DMN was considered as a memory-based simulation system, serving to piece together materials from one's past experience to construct new scenes or context, which can be self-projected into for evaluation, prospection, and mentalizing (Buckner and Carroll, 2007; Hassabis and Maguire, 2007; Schacter et al., 2007; Buckner et al., 2008). It has been discussed that semantic processing is a necessary component underlying such processes (Binder et al., 1999, 2009; Binder and Desai, 2011; Binder, 2012).

We wish to bring attention to another intriguing aspect of the DMN: many of its constituent regions are where information from multiple modalities converges. Using a "stepwise functional connectivity" approach to trace information pathways from unimodal regions to higher-order convergence zones, areas in the DMN were found to be the final stable state where information pathways from all modalities reach (Sepulcre et al., 2012). Applying a "parametric modulation" method to decompose the activation of a word into effects of multiple modality-specific attributes, areas where all the attribute effects overlapped largely fall in the DMN (Fernandino et al., 2016) (Figure 2B, right). This evidence suggest that this system is likely to support the integration of simulation-based multimodal experiential representation. Using the earlier "Beijing" example, we can use our experience to construct multimodal scenarios about what "Beijing" entails, e.g., the views of the Forbidden City, the taste or smell of a Beijing roast duck, or the rhotic vowels of the Beijing dialect. As concepts acquired from rich personal experience can be more automatically instantiated through this approach, the DMN is more strongly activated by concrete
(Binder et al., 2005; Sabsevitz et al., 2005; Wang et al., 2010; Hoffman et al., 2015), famous (Sugiura et al., 2006; Wang et al., 2016), and personal (Sugiura et al., 2006; Renoult et al., 2012) concepts, in contrast with abstract, common, and general concepts, respectively. Patients with Alzheimer's disease or mild cognitive impairment in which the DMN is usually compromised tended to recall past events divested of rich sensory-perceptual imagery (Irish et al., 2011), and show deficits of knowledge of famous people and their physical features (Borg et al., 2010).

However, the DMN is neither sufficient nor necessary for all semantic tasks. Unlike damage to the PSN (see below), damage to brain areas of the DMN in patients with Alzheimer's disease (Nestor et al., 2006) or in patients suffering a stroke with lesions encompassing the posterior cingulate cortices (Leech and Sharp, 2014) or the parietooccipital cortex (Berryhill et al., 2007) seems not to cause severe deficits in semantic tasks that mostly probe association or function knowledge. We thus contend that the DMN hosts the aspects of semantic knowledge that are tightly related to multimodal experiences and is not necessary for those tasks that do not require the retrieval of specific attributes based on such experiences (e.g., associating "Beijing" with "China" does not require retrieval of the specific location or landscape of Beijing, and "fox" with "shrewdness" does not require retrieval of what a fox's ears look like).

\section{The PSN: The Language-Supported Semantic System}

This module (green nodes in Figure 2A, middle) includes the entire length of the left middle temporal gyrus, the ventral part of the left IFG, and the junction area of the left posterior temporal and inferior parietal lobes (the left temporoparietal junction). A common characteristic of these regions is that they together fit well with the language network (Figure 2B, middle) (Friederici, 2011), which shows selective activation by sentences in contrast to nonword lists and not by multiple non-linguistic tasks, such as, arithmetic, working memory, cognitive control, or music (Fedorenko et al., 2011). For semantic processing, the left ventral IFG and the left temporal cortex in this module were consistently found to be more strongly activated by abstract and idiomatic concepts with meanings that presumably rely heavily on linguistic associations (Hoffman, 2015) than by concrete (Binder et al., 2005; Sabsevitz et al., 2005; Wang et al., 2010; Hoffman et al., 2015) or literal (Lauro et al., 2008; Boulenger et al., 2009) terms. Intriguingly, lesions or atrophies in regions of this system affect semantic comprehension in not only verbal but also nonverbal tasks using picture, sound or motion as inputs, e.g., the anterior temporal cortex (Bozeat et al., 2000; Mummery et al., 2000; Garrard and Carroll, 2006; Jefferies and Lambon Ralph, 2006; Robson et al., 2012), the left posterior temporal and temporoparietal cortices (Jefferies and Lambon Ralph, 2006; Corbett et al., 2009; Robson et al., 2012; Thompson et al., 2015). Transcranial magnetic stimulation (TMS) to the left (ATL) (Pobric et al., 2007, 2010) and the left posterior middle temporal gyrus (pMTG) (Hoffman et al., 2012) impedes semantic performances in both verbal and nonverbal tasks without affecting non-semantic tasks of comparable difficulty. 
What kind of function would be relevant for linguistic processing and for semantic processing in both verbal and nonverbal tasks? We postulate that this module supports (amodal) semantic representation that is embedded in the language system. Given the paucity of research on languagerelated dimensions in the neural semantic space, the exact nature, content, or format of representation that is supported by the PSN remains unknown. The point here is that the identification of a PSN module (segregated from the DMN) in the semantic brain network suggests a natural candidate system for a kind of representation distinct from experientialbased representations. There has been much discussion in the cognitive, psycholinguistic and artificial intelligence fields about how linguistic contexts (e.g., word association, word order, and syntactic structure) contribute to representing meaning (Landauer and Dumais, 1997; Burgess, 1998; Jones and Mewhort, 2007; Barsalou et al., 2008; Dove, 2009, 2010; Vigliocco et al., 2009; Mikolov et al., 2013a, 2014), and relevant hypotheses should be articulated for testing against neural responses in the PSN. One simple possibility is that the occurrence patterns in natural language differ from the objects and events associations in the real-word scenes at least to some extent, and such language-occurrences modulate the experience-based relations among concepts and create new types of relations. That is, the specific association patterns due to the linguistic contexts, among lexical representations and/or semantic representation in the DMN system, may give rise to information that is part of the semantic representation. There are two important points to note. First, while the symbolic accounts of semantic representation may satisfy this description, the representational format in the Module PSN is not necessarily amodal symbolic. Second, there is a long debate about the necessity of having "lexicalized concepts" being different from "prelinguistic concepts" (Caramazza, 1997; Levelt et al., 1999; Vigliocco and Vinson, 2007). In the spirit of parsimony, we do not think having a separate lexicalized concepts here are necessary. The semantic information supported by the language system could be coded in the association patterns of lexical representations themselves, which points to "prelinguistic" concepts (the experiential representations in the Module DMN). Importantly, such language-supported knowledge constitutes an integral aspect of semantics (consider the knowledge given by "Beijing is the capital of China" for "Beijing"), and disrupting this module would lead to impairment for semantic tasks requiring this type of knowledge in not only verbal but also nonverbal semantic tasks.

\section{The IFPN: The Semantic Control System}

This module (blue nodes in Figure 2A, middle), including the dorsal part of the left IFG, the IPS, and a region in the posterior inferior temporal lobe. It is largely similar to the left hemisphere part of a broader bilateral frontoparietal control system revealed by whole-brain intrinsic functional connectivity analyses (Vincent et al., 2008; Power et al., 2011; Yeo et al., 2011). The broader frontoparietal control network acts as a flexible hub (Cole et al., 2013), offering rapid adaptive coordination of other functional systems in a task- and time-appropriate fashion (Dosenbach et al., 2008). While the right part of this network is involved in sensorimotor-related control (Levy and Wagner, 2011; Harel et al., 2014), the left one is more engaged in the conceptual and linguistic domains (Noonan et al., 2013; Harel et al., 2014). Compared to the FPN that has been referred to a multi-demand system that is activated during a wide variety of demanding cognitive tasks (Duncan, 2010; Fedorenko et al., 2013), the FPN that is most consistently associated with semantic control is more left-lateralized with its frontal part being more posterior and inferior.

The proposal that regions in this module serve a control role in semantic cognition, i.e., semantic control, has been discussed in depth in recent reviews (Jefferies, 2013; Lambon Ralph et al., 2016). We will not reiterate all the relevant empirical evidence here but will refer to a few lines of representative evidence (Table 1). Meta-analyses show that brain areas in the IFPN are more strongly activated by semantic tasks requiring greater semantic control (Noonan et al., 2013) (Figure 2B, right). Attribute semantic tasks, which require attention on memory images of specific attributes (e.g., color, shape, manipulation), induce stronger activation in this module, in contrast to semantic tasks, which do not (Badre et al., 2005; Davey et al., 2016). Lesions extending to the IFPN lead to so-called "semantic access deficits" (Mirman and Britt, 2014), with sensitivity to the semantic distance and the strength of competitors in semantic association tasks (Noonan et al., 2010), refractory effects (Jefferies et al., 2007; Thompson et al., 2015), and item or task inconsistency across different semantic tasks (Jefferies and Lambon Ralph, 2006; Corbett et al., 2009; Robson et al., 2012). TMS to the triangular part of the left IFG selectively disrupts semantic tasks with higher executive demands without affecting those with low demand or non-semantic tasks and to the left IPS disrupts performance in attribute semantic tasks (Whitney et al., 2011, 2012; KriegerRedwood and Jefferies, 2014). In the context of the threemodule structure, this system "controls" the semantic content represented in the other two modules-retrieving the specific experiential attributes in the DMN and the language-based knowledge in the PSN-according to current task demands. Regions in this system are more strongly activated by semantic tasks than by other tasks that presumably also need control (Binder et al., 2009), either because semantic processing requires stronger/more complex controls or because these regions are more strongly connected with other semantic representation components and are more visible in semantic tasks.

\section{INTEGRATED HUBS, INTEGRATED SEMANTIC COMPONENTS}

The three modules must be integrated for a given semantic task. When we hear the word "Beijing" or see a picture of Beijing in various linguistic or real-world contexts, multiple aspects of semantic knowledge and the control systems are activated to achieve understanding. How are these three modules integrated? The network analysis identified a series of connector hub regions that are important in linking the three modules discussed above (Figure 2A, right) (Xu et al., 2016): the left ATL linking Modules PSN and DMN, the left pMTG linking Modules PSN and IFPN, 
the left posterior intraparietal sulcus (pIPS) linking Modules DMN and IFPN, and the left AG and the border areas of the superior and middle frontal gyri (left SFG/MFG) linking all three brain systems. Note that these regions were also discussed above in the three modules; all regions are assigned to a module, even regions with relatively evenly distributed connections with multiple modules (i.e., connector hubs). These regions, especially the ATL (Patterson et al., 2007; Lambon Ralph et al., 2016), the pMTG (Wei et al., 2012; Davey et al., 2016), and the AG (Schwartz et al., 2011; Bonner et al., 2013; Seghier, 2013; Price et al., 2015), have been considered to be the "hub" regions of the semantic system, motivated by various types of evidence about their importance in semantic processing. Based on the literature, multiple types of semantic functions, including the hypothesized ones of the corresponding networks they link, have been reported in these regions (Table 1).

The connectivity-based findings reviewed here provide direct empirical evidence for their (connector-) "hub" status, the definition of which is based on the connectivity patterns (Guimera and Amaral, 2005; van den Heuvel and Sporns, 2013), and revealed that they differ in terms of the systems they connect. For instance, the ATL is where multimodal experiential representation and language-supported representation meet, whereas the pIPS and the pMTG are where the control system interacts with the experiential and language-supported representations, respectively. These findings that derived from the topological patterns of these hub regions are in accordance with some previous notions about these regions that was inferred from regional activation patterns, e.g., that the ATL is the "transmodal" site between experiential and language-supported representations (Rogers et al., 2004; Patterson et al., 2007; Visser et al., 2010; Lambon Ralph, 2014; Rice et al., 2015), the IPS is associated with top-down attention to memory images (Cabeza et al., 2008), and the pMTG is the area for "controlled semantic retrieval” (Badre et al., 2005; Schwartz et al., 2011; Davey et al., 2015).

Notably, while the connectivity profiles suggest that they are likely to be the sites where different components of semantic processing are integrated, there are at least two possibilities about whether and how they actually merge. One is that they simply host adjacent yet different sub-regions belonging to different networks and with distinct functionalities. Another possibility is that they perform a similar function to different inputs within different tasks or some type of higher-order computations that merge the functions of multiple modules. In light of the network structure, studies on the nature of representation and processing supported by these regions should take into consideration the functions of the multiple networks they merge.

\section{A TRI-NETWORK NEUROCOGNITIVE MODEL OF SEMANTIC PROCESSING}

Our review of recent brain network studies on semantic processing and consideration of previous region-based results in light of these new findings lead to the proposal of a trinetwork neurocognitive model of human semantic processing
(Figure 3): the widely distributed semantic regions are wired into three separate neural networks, which are likely to support three different cognitive components of semantic processing. The DMN serves as the multimodal experiential system, where experience-based knowledge across multiple modalities is integrated (e.g., the integration of various types of experiences one has with Beijing). The left PSN serves as the non-experiential system, where semantic content being supported by linguistic contexts are represented (e.g., "Beijing is the capital of China"). The IFPN serves as the semantic control system, acting on the other two modules for the retrieval of semantic knowledge in a task- and time-appropriate fashion. Semantic processing entails the coordination of these functional modules, which is likely to be achieved via a series of connector hubs in the ATL, the PMTG, the pIPS, the AG, and the SFG/MFG.

This tri-network model is mainly motivated by the modular and hub structure of the widely distributed semantic network and shares several key points with previous semantic neurocognitive models. The role of the ATL in binding various modalities (verbal and non-verbal) converges with the "hub and spoke" model as well as its recent update-the controlled semantic cognition model (Patterson et al., 2007; Lambon Ralph et al., 2016). The relevance of this distributed system in representing abstraction of modality-specific attributes/experiences is shared by the embodied-abstraction model (Binder and Desai, 2011). The distinction between representation and control is also well in line with these recent models (Binder and Desai, 2011; Jefferies, 2013; Lambon Ralph et al., 2016).

However, there are several important differences. Within representation, our current framework entails two segregated systems: multimodal experiential content in the DMN and language-supported content in the PSN. Both are "abstracted" away from modality-specific "embodiment," but the degree of abstraction and the principle of abstraction are likely to differ between these two systems-one originated from realworld experience and one from language. In the DMN, the sensory, motor, and affective inputs from multiple modalityspecific systems converge together to capture high-order conceptual representations (e.g., taxonomic categories or a

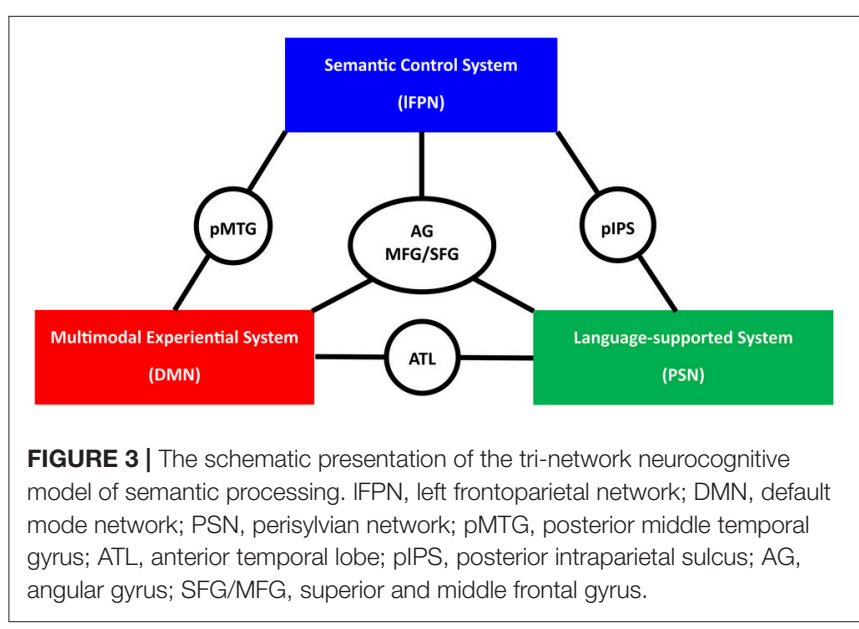


whole event), which best fill the role of the "crossmodal conjunctive representation" proposed by Binder (2016). In the PSN, the meaning is supported by language contexts. The previous neuroanatomical semantic models have not articulated such potential differences between language-supported and experience-based semantic representations. In some models, only modality/property-specific representations were considered (Martin, 2016). In the hub-and-spoke/controlled semantic cognition model, language is just one modality of processing in parallel to other modalities (e.g., vision, sound, and valence) (Patterson et al., 2007; Lambon Ralph et al., 2016). In the embodied-abstraction model, abstraction happens from the modality-specific representations (Binder et al., 2009; Binder and Desai, 2011). Our framework incorporates evidence both from the network structure and regional studies using lesion and neuro-activation approaches, highlights the two distinct brain systems for two different types of semantic representation, which is more similar to the dual coding cognitive models of meaning (Paivio, 1986; Barsalou et al., 2008; Mahon and Caramazza, 2008; Dove, 2009, 2010; Vigliocco et al., 2009; Zwaan, 2014; Reilly et al., 2016), and provides specific dissociable target brain systems as the corresponding neural bases. A series of hub regions are also explicitly postulated to integrate the two kinds of semantic representation and to interact with the control system.

\section{FUTURE PERSPECTIVES}

This tri-network model frames several lines of new questions about the brain basis of semantics. The central point is that from any of the various approaches, instead of studying the functionality and mechanisms of widely distributed cortical regions implicated in semantic processing individually, it would be more productive to study them in the contexts of the three sub-systems, examining both the modules as whole units and the roles of specific constituents (regions and connections). A few examples are outlined here. First, regarding different kinds of semantic processing, it remains to be understood how the connectivity pattern across different modules is configured according to different types of semantic tasks (e.g., with different semantic contents and difficulty levels). Would the configuration of connectivity patterns complement the findings of cortical representations? Specifically, would the connectivity within a particular module be specifically strengthened when the task involved more of the corresponding semantic contents (e.g., the Module PSN in a task that requires to process abstract concepts)? Would the connectivity between the Module FPN and the other two modules be strengthened when the task was more difficult? Second, about the

\section{REFERENCES}

Acosta-Cabronero, J., Patterson, K., Fryer, T.D., Hodges, J. R., Pengas, G., Williams, G. B., et al. (2011). Atrophy, hypometabolism and white matter abnormalities in semantic dementia tell a coherent story. Brain 134, 2025-2035. doi: 10.1093/brain/awr119 different types of semantic content and encoding mechanisms (experiential vs. those supported by linguistic contexts), the DMN and the PSN modules in the semantic system provide the candidate target brain systems to test their distinctions and interactions. Cognitive models built from experiencebased attributes and those from various natural language processing models, e.g., the Latent Semantic Analysis (Landauer and Dumais, 1997) or the neural network models such as, word2vec (Mikolov et al., 2013a, 2014), could be compared against neural activity patterns in these two neural modules. Will activity pattern and/or connectivity pattern across the DMN and the PSN modules correlate relatively more strongly with the semantic space generated according to experiential and the linguistic contextual models, respectively? Third, from a developmental perspective, it would be intriguing to see during semantic knowledge acquisition whether the neural representational patterns of different sub-systems are modulated by corresponding types of experience (linguistic vs. experimental). Finally, new questions emerge about the nature of computation at the connector-hub regions, i.e., how information across multiple modules is integrated. Do the hub regions simply host sub-regions with distinct functionalities (exhibiting distinct neural representational pattern) or do they perform some type of higher-order computation that merges the functions of multiple modules (exhibiting high-order neural representational patterns)?

\section{AUTHOR CONTRIBUTIONS}

All authors listed have made a substantial, direct and intellectual contribution to the work, and approved it for publication.

\section{FUNDING}

The authors are funded by the National Key Basic Research Program of China (2013CB837300 and 2014CB846100), National Natural Science Foundation of China (31671128, 91432115 and 81620108016), National Program for Special Support of Top-notch Young Professionals (YB), Beijing Brain Project (Z16110100020000, Z161100000216125), and the Fundamental Research Funds for the Central Universities (2017XTCX04).

\section{ACKNOWLEDGMENTS}

We are grateful to Xiaoying Wang, Xiaosha Wang, and Huichao Yang for helpful discussions and comments on an earlier version of the manuscript. We thank Dr. Evelina Fedorenko, Leonardo Fernandino, and Matthew A. Lambon Ralph for permission to use their figures in our paper. 
Badre, D., Poldrack, R. A., Pare-Blagoev, E. J., Insler, R. Z., and Wagner, A. D. (2005). Dissociable controlled retrieval and generalized selection mechanisms in ventrolateral prefrontal cortex. Neuron 47, 907-918. doi: 10.1016/j.neuron.2005.07.023

Barsalou, L. W., Santos, A., Simmons, W. K., and Wilson, C. D. (2008). "Language and simulation in conceptual processing," in Symbols, Embodiment, and Meaning, eds M. De Vega, A. Glenberg, and A. Graesser (Oxford, UK: Oxford University Press), 245-283. doi: 10.1093/acprof:oso/9780199217274.003.0013

Basser, P. J., Mattiello, J., and Lebihan, D. (1994). MR diffusion tensor spectroscopy and imaging. Biophys. J. 66:259. doi: 10.1016/S0006-3495(94)80775-1

Berryhill, M. E., Phuong, L., Picasso, L., Cabeza, R., and Olson, I. R. (2007). Parietal lobe and episodic memory: bilateral damage causes impaired free recall of autobiographical memory. J. Neurosci. 27, 14415-14423. doi: 10.1523/JNEUROSCI.4163-07.2007

Binder, J. R. (2012). Task-induced deactivation and the "resting" state. Neuroimage 62, 1086-1091. doi: 10.1016/j.neuroimage.2011.09.026

Binder, J. R. (2016). In defense of abstract conceptual representations. Psychon. Bull. Rev. 23, 1096-1108. doi: 10.3758/s13423-015-0909-1

Binder, J. R., and Desai, R. H. (2011). The neurobiology of semantic memory. Trends Cogn. Sci. 15, 527-536. doi: 10.1016/j.tics.2011.10.001

Binder, J. R., Desai, R. H., Graves, W. W., and Conant, L. L. (2009). Where is the semantic system? A critical review and meta-analysis of 120 functional neuroimaging studies. Cereb. Cortex 19, 2767-2796. doi: 10.1093/cercor/bhp055

Binder, J. R., Frost, J. A., Hammeke, T. A., Bellgowan, P. S., Rao, S. M., and Cox, R. W. (1999). Conceptual processing during the conscious resting state. A functional MRI study. J. Cogn. Neurosci. 11, 80-95. doi: $10.1162 / 089892999563265$

Binder, J. R., Westbury, C. F., McKiernan, K. A., Possing, E. T., and Medler, D. A. (2005). Distinct brain systems for processing concrete and abstract concepts. J. Cogn. Neurosci. 17, 905-917. doi: 10.1162/0898929054021102

Binney, R. J., Parker, G. J., and Ralph, M. A. L. (2012). Convergent connectivity and graded specialization in the rostral human temporal lobe as revealed by diffusion-weighted imaging probabilistic tractography. J. Cogn. Neurosci. 24, 1998-2014. doi: 10.1162/jocn_a_00263

Biswal, B., Yetkin, F. Z., Haughton, V. M., and Hyde, J.S. (1995). Functional connectivity in the motor cortex of resting human brain using echo-planar MRI. Magn. Reson. Med. 34, 537-541. doi: 10.1002/mrm.1910340409

Bonner, M. F., Peelle, J. E., Cook, P. A., and Grossman, M. (2013). Heteromodal conceptual processing in the angular gyrus. Neuroimage 71, 175-186. doi: 10.1016/j.neuroimage.2013.01.006

Borg, C., Thomas-Antérion, C., Bogey, S., Davier, K., and Laurent, B. (2010). Visual imagery processing and knowledge of famous names in Alzheimer's disease and MCI. Aging Neuropsychol. Cogn. 17, 603-614. doi: 10.1080/13825585.2010.481357

Boulenger, V., Hauk, O., and Pulvermüller, F. (2009). Grasping ideas with the motor system: semantic somatotopy in idiom comprehension. Cereb. Cortex 19, 1905-1914. doi: 10.1093/cercor/bhn217

Bozeat, S., Lambon Ralph, M. A., Patterson, K., Garrard, P., and Hodges, J. R. (2000). Non-verbal semantic impairment in semantic dementia. Neuropsychologia 38, 1207-1215. doi: 10.1016/S0028-3932(00)00034-8

Buckner, R. L., and Carroll, D. C. (2007). Self-projection and the brain. Trends Cogn. Sci. 11, 49-57. doi: 10.1016/j.tics.2006.11.004

Buckner, R. L., Andrews-Hanna, J. R., and Schacter, D. L. (2008). The brain's default network: anatomy, function, and relevance to disease. Ann. N. Y. Acad. Sci. 1124, 1-38. doi: 10.1196/annals.1440.011

Bullmore, E., and Sporns, O. (2009). Complex brain networks: graph theoretical analysis of structural and functional systems. Nat. Rev. Neurosci. 10, 186-198. doi: $10.1038 / \mathrm{nrn} 2575$

Burgess, C. (1998). From simple associations to the building blocks of language: modeling meaning in memory with the HAL model. Behav. Res. Methods Instrum. Comput. 30, 188-198. doi: 10.3758/BF03200643

Cabeza, R., Ciaramelli, E., Olson, I. R., and Moscovitch, M. (2008). The parietal cortex and episodic memory: an attentional account. Nat. Rev. Neurosci. 9, 613-625. doi: 10.1038/nrn2459

Caramazza, A. (1997). How many levels of processing are there in lexical access? Cogn. Neuropsychol. 14, 177-208. doi: 10.1080/026432997381664

Chai, X. J., Castaón, A. N., Öngür, D., and Whitfield-Gabrieli, S. (2012). Anticorrelations in resting state networks without global signal regression. Neuroimage 59, 1420-1428. doi: 10.1016/j.neuroimage.2011. 08.048

Cole, M. W., Reynolds, J. R., Power, J. D., Repovs, G., Anticevic, A., and Braver, T. S. (2013). Multi-task connectivity reveals flexible hubs for adaptive task control. Nat. Rev. Neurosci. 16, 1348-1355. doi: 10.1038/nn.3470

Corbett, F., Jefferies, E., Ehsan, S., and Lambon Ralph, M.A. (2009). Different impairments of semantic cognition in semantic dementia and semantic aphasia: evidence from the non-verbal domain. Brain 132, 2593-2608. doi: 10.1093/brain/awp146

Davey, J., Cornelissen, P. L., Thompson, H. E., Sonkusare, S., Hallam, G., Smallwood, J., et al. (2015). Automatic and controlled semantic retrieval: TMS reveals distinct contributions of posterior middle temporal gyrus and angular gyrus. J. Neurosci. 35, 15230-15239. doi: 10.1523/JNEUROSCI.4705-14.2015

Davey, J., Thompson, H. E., Hallam, G., Karapanagiotidis, T., Murphy, C., De Caso, I., et al. (2016). Exploring the role of the posterior middle temporal gyrus in semantic cognition: integration of anterior temporal lobe with executive processes. Neuroimage 137, 165-177. doi: 10.1016/j.neuroimage.2016.05.051

Dosenbach, N. U., Fair, D. A., Cohen, A. L., Schlaggar, B. L., and Petersen, S. E. (2008). A dual-networks architecture of top-down control. Trends Cogn. Sci. 12, 99-105. doi: 10.1016/j.tics.2008.01.001

Dove, G. (2009). Beyond perceptual symbols: a call for representational pluralism. Cognition 110, 412-431. doi: 10.1016/j.cognition.2008.11.016

Dove, G. (2010). On the need for embodied and dis-embodied cognition. Front. Psychol. 1:242. doi: 10.3389/fpsyg.2010.00242

Duffau, H., Gatignol, P., Mandonnet, E., Peruzzi, P., Tzourio-Mazoyer, N., and Capelle, L. (2005). New insights into the anatomo-functional connectivity of the semantic system: a study using cortico-subcortical electrostimulations. Brain 128, 797-810. doi: 10.1093/brain/awh423

Duncan, J. (2010). The multiple-demand (MD) system of the primate brain: mental programs for intelligent behaviour. Trends Cogn. Sci. 14, 172-179. doi: 10.1016/j.tics.2010.01.004

Fang, Y., Han, Z., Zhong, S., Gong, G., Song, L., Liu, F., et al. (2015). The semantic anatomical network: evidence from healthy and brain-damaged patient populations. Hum. Brain Mapp. 36, 3499-3515. doi: 10.1002/hbm. 22858

Fedorenko, E., Behr, M. K., and Kanwisher, N. (2011). Functional specificity for high-level linguistic processing in the human brain. Proc. Natl. Acad. Sci. U.S.A. 108, 16428-16433. doi: 10.1073/pnas.1112937108

Fedorenko, E., Duncan, J., and Kanwisher, N. (2013). Broad domain generality in focal regions of frontal and parietal cortex. Proc. Natl. Acad. Sci. U.S.A. 110, 16616-16621. doi: 10.1073/pnas.1315235110

Feng, G., Chen, Q., Zhu, Z., and Wang, S. (2015). Separate brain circuits support integrative and semantic priming in the human language system. Cereb. Cortex 26, 3169-3182. doi: 10.1093/cercor/bhv148

Fernandino, L., Binder, J. R., Desai, R. H., Pendl, S. L., Humphries, C. J., Gross, W. L., et al. (2016). Concept representation reflects multimodal abstraction: a framework for embodied semantics. Cereb. Cortex 26, 2018-2034. doi: 10.1093/cercor/bhv020

Fortunato, S. (2010). Community detection in graphs. Phys. Rep. 486, 75-174. doi: 10.1016/j.physrep.2009.11.002

Fox, M. D., Snyder, A. Z., Vincent, J. L., Corbetta, M., Van Essen, D. C., and Raichle, M. E. (2005). The human brain is intrinsically organized into dynamic, anticorrelated functional networks. Proc. Nat. Acad. Sci. U.S.A. 102, 9673-9678. doi: $10.1073 /$ pnas. 0504136102

Friederici, A. D. (2011). The brain basis of language processing: from structure to function. Physiol. Rev. 91, 1357-1392. doi: 10.1152/physrev.00006.2011

Garrard, P., and Carroll, E. (2006). Lost in semantic space: a multi-modal, nonverbal assessment of feature knowledge in semantic dementia. Brain 129, 1152-1163. doi: 10.1093/brain/awl069

Guimera, R., and Amaral, L. N. (2005). Functional cartography of complex metabolic networks. Nature 433, 895-900. doi: 10.1038/nature03288

Han, Z., Ma, Y., Gong, G., He, Y., Caramazza, A., and Bi, Y. (2013). White matter structural connectivity underlying semantic processing: evidence from brain damaged patients. Brain 136, 2952-2965. doi: 10.1093/brain/awt205

Harel, A., Kravitz, D. J., and Baker, C. I. (2014). Task context impacts visual object processing differentially across the cortex. Proc. Natl. Acad. Sci. U.S.A. 111, E962-E971. doi: 10.1073/pnas.1312567111

Hartwigsen, G., Weigel, A., Schuschan, P., Siebner, H.R., Weise, D., Classen, J., et al. (2015). Dissociating parieto-frontal networks for phonological and 
semantic word decisions: a condition-and-perturb TMS study. Cereb. Cortex 26, 2590-2601. doi: 10.1093/cercor/bhv092

Hassabis, D., and Maguire, E.A. (2007). Deconstructing episodic memory with construction. Trends Cogn. Sci. 11, 299-306. doi: 10.1016/j.tics.2007.05.001

He, Y., and Evans, A. (2010). Graph theoretical modeling of brain connectivity. Curr. Opin. Neurobiol. 23, 341-350. doi: 10.1097/WCO.0b013e32833aa567

He, Y., Wang, J., Wang, L., Chen, Z. J., Yan, C., Yang, H., et al. (2009). Uncovering intrinsic modular organization of spontaneous brain activity in humans. PLoS ONE 4:e5226. doi: 10.1371/journal.pone.0005226

Henson, R. (2005). What can functional neuroimaging tell the experimental psychologist? Q. J. Exp. Psychol. A 58, 193-233. doi: 10.1080/02724980443 000502

Hoffman, P. (2015). The meaning of "life" and other abstract words: insights from neuropsychology. J. Neuropsychol. 10, 317-343. doi: 10.1111/jnp.12065

Hoffman, P., Binney, R. J., and Lambon Ralph, M. A. (2015). Differing contributions of inferior prefrontal and anterior temporal cortex to concrete and abstract conceptual knowledge. Cortex 63, 250-266. doi: 10.1016/j.cortex.2014.09.001

Hoffman, P., Pobric, G., Drakesmith, M., and Lambon Ralph, M. A. (2012). Posterior middle temporal gyrus is involved in verbal and non-verbal semantic cognition: evidence from rTMS. Aphasiology 26, 1119-1130. doi: 10.1080/02687038.2011.608838

Humphreys, G. F., Hoffman, P., Visser, M., Binney, R. J., and Lambon Ralph, M. A. (2015). Establishing task- and modality-dependent dissociations between the semantic and default mode networks. Proc. Natl. Acad. Sci. U.S.A. 112, 7857-7862. doi: $10.1073 /$ pnas. 1422760112

Irish, M., Lawlor, B. A., O'mara, S. M., and Coen, R. F. (2011). Impaired capacity for autonoetic reliving during autobiographical event recall in mild Alzheimer's disease. Cortex 47, 236-249. doi: 10.1016/j.cortex.2010.01.002

Jackson, R. L., Hoffman, P., Pobric, G., and Ralph, M. L. (2016). The semantic network at work and rest: differential connectivity of anterior temporal lobe subregions. J. Neurosci. 36, 1490-1501. doi: 10.1523/JNEUROSCI.2999-15.2016

Jefferies, E. (2013). The neural basis of semantic cognition: converging evidence from neuropsychology, neuroimaging and TMS. Cortex 49, 611-625. doi: 10.1016/j.cortex.2012.10.008

Jefferies, E., and Lambon Ralph, M.A. (2006). Semantic impairment in stroke aphasia versus semantic dementia: a case-series comparison. Brain 129, 2132-2147. doi: 10.1093/brain/awl153

Jefferies, E., Baker, S. S., Doran, M., and Ralph, M. L. (2007). Refractory effects in stroke aphasia: a consequence of poor semantic control. Neuropsychologia 45, 1065-1079. doi: 10.1016/j.neuropsychologia.2006.09.009

Jones, M. N., and Mewhort, D. J. (2007). Representing word meaning and order information in a composite holographic lexicon. Psychol. Rev. 114:1. doi: 10.1037/0033-295X.114.1.1

Krieger-Redwood, K., and Jefferies, E. (2014). TMS interferes with lexicalsemantic retrieval in left inferior frontal gyrus and posterior middle temporal gyrus: evidence from cyclical picture naming. Neuropsychologia 64, 24-32. doi: 10.1016/j.neuropsychologia.2014.09.014

Lambon Ralph, M. A. (2014). Neurocognitive insights on conceptual knowledge and its breakdown. Philos. Trans. R. Soc. Lond. B 369:20120392. doi: 10.1098/rstb.2012.0392

Lambon Ralph, M. A., Jefferies, E., Patterson, K., and Rogers, T. T. (2016). The neural and computational bases of semantic cognition. Nat. Rev. Neurosci. 18, 42-55. doi: 10.1038/nrn.2016.150

Landauer, T. K., and Dumais, S. T. (1997). A solution to Plato's problem: the latent semantic analysis theory of acquisition, induction, and representation of knowledge. Psychol. Rev. 104:211. doi: 10.1037/0033-295X.104.2.211

Lauro, L. J. R., Tettamanti, M., Cappa, S. F., and Papagno, C. (2008). Idiom comprehension: a prefrontal task? Cereb. Cortex 18, 162-170. doi: $10.1093 /$ cercor/bhm042

Leech, R., and Sharp, D. J. (2014). The role of the posterior cingulate cortex in cognition and disease. Brain 137, 12-32. doi: 10.1093/brain/awt162

Levelt, W. J., Roelofs, A., and Meyer, A. S. (1999). A theory of lexical access in speech production. Behav. Brain Sci. 22, 1-38. doi: $10.1017 /$ S0140525X99001776

Levy, B. J., and Wagner, A. D. (2011). Cognitive control and right ventrolateral prefrontal cortex: reflexive reorienting, motor inhibition, and action updating. Ann. N. Y. Acad. Sci. 1224, 40-62. doi: 10.1111/j.1749-6632.2011.05958.x
Mahon, B. Z., and Caramazza, A. (2008). A critical look at the embodied cognition hypothesis and a new proposal for grounding conceptual content. J. Physiol. 102, 59-70. doi: 10.1016/j.jphysparis.2008.03.004

Martin, A. (2016). GRAPES-Grounding representations in action, perception, and emotion systems: how object properties and categories are represented in the human brain. Psychon. Bull. Rev, 23, 1-12. doi: 10.3758/s13423-015-0842-3

Mikolov, T., Chen, K., Corrado, G., and Dean, J. (2013a). Efficient estimation of word representations in vector space. arXiv preprint arXiv:1301.3781.

Mikolov, T., Sutskever, I., Chen, K., Corrado, G. S., and Dean, J. (2014). "Distributed representations of words and phrases and their compositionality," in Advances in Neural Information Processing Systems, eds C. J. C. Burges, L. Bottou, M. Welling, Z. Ghahramani, and K. Q. Weinberger (Red Hook, NY: Curran Associates, Inc.), 3111-3119.

Mirman, D., and Britt, A. E. (2014). What we talk about when we talk about access deficits. Philos. Trans. R. Soc. Lond. B 369, 20120388. doi: $10.1098 /$ rstb.2012.0388

Mummery, C. J., Patterson, K., Price, C., Ashburner, J., Frackowiak, R., and Hodges, J. R. (2000). A voxel-based morphometry study of semantic dementia: relationship between temporal lobe atrophy and semantic memory. Ann. Neurol. 47, 36-45. doi: 10.1002/1531-8249(200001)47:1<36::AID-ANA8 > 3.0.CO;2-L

Nestor, P. J., Fryer, T. D., and Hodges, J. R. (2006). Declarative memory impairments in Alzheimer's disease and semantic dementia. Neuroimage 30, 1010-1020. doi: 10.1016/j.neuroimage.2005.10.008

Noonan, K. A., Jefferies, E., Corbett, F., and Ralph, M. L. (2010). Elucidating the nature of deregulated semantic cognition in semantic aphasia: evidence for the roles of prefrontal and temporo-parietal cortices. J. Cogn. Neurosci. 22, 1597-1613. doi: 10.1162/jocn.2009.21289

Noonan, K. A., Jefferies, E., Visser, M., and Ralph, M. L. (2013). Going beyond inferior prefrontal involvement in semantic control: evidence for the additional contribution of dorsal angular gyrus and posterior middle temporal cortex. $J$. Cogn. Neurosci. 25, 1824-1850. doi: 10.1162/jocn_a_00442

Paivio, A. (1986). Mental Representation: A Dual-Coding Approach. New York, NY: Oxford University Press.

Pascual, B., Masdeu, J. C., Hollenbeck, M., Makris, N., Insausti, R., Ding, S.-L., et al. (2013). Large-scale brain networks of the human left temporal pole: a functional connectivity MRI study. Cereb. Cortex 25, 680-702. doi: 10.1093/cercor/bht260

Passingham, R. E., Stephan, K. E., and Kötter, R. (2002). The anatomical basis of functional localization in the cortex. Nat. Rev. Neurosci. 3, 606-616. doi: $10.1038 / \mathrm{nrn} 893$

Patterson, K., Nestor, P. J., and Rogers, T. T. (2007). Where do you know what you know? The representation of semantic knowledge in the human brain. Nat. Rev. Neurosci. 8, 976-987. doi: 10.1038/nrn2277

Pobric, G., Jefferies, E., and Ralph, M. L. (2007). Anterior temporal lobes mediate semantic representation: mimicking semantic dementia by using rTMS in normal participants. Proc. Natl. Acad. Sci. U.S.A. 104, 20137-20141. doi: 10.1073/pnas.0707383104

Pobric, G., Jefferies, E., and Ralph, M. A. L. (2010). Amodal semantic representations depend on both anterior temporal lobes: evidence from repetitive transcranial magnetic stimulation. Neuropsychologia 48, 1336-1342. doi: 10.1016/j.neuropsychologia.2009.12.036

Poldrack, R. A. (2006). Can cognitive processes be inferred from neuroimaging data? Trends Cogn. Sci. 10, 59-63. doi: 10.1016/j.tics.2005.12.004

Power, J. D., Cohen, A. L., Nelson, S. M., Wig, G. S., Barnes, K. A., Church, J. A., et al. (2011). Functional network organization of the human brain. Neuron 72, 665-678. doi: 10.1016/j.neuron.2011.09.006

Power, J. D., Schlaggar, B. L., Lessov-Schlaggar, C. N., and Petersen, S. E. (2013). Evidence for hubs in human functional brain networks. Neuron 79, 798-813. doi: 10.1016/j.neuron.2013.07.035

Price, A. R., Bonner, M. F., Peelle, J. E., and Grossman, M. (2015). Converging evidence for the neuroanatomic basis of combinatorial semantics in the angular gyrus. J. Neurosci. 35, 3276-3284. doi: 10.1523/JNEUROSCI.344614.2015

Price, C. J., and Friston, K. J. (2005). Functional ontologies for cognition: the systematic definition of structure and function. Cogn. Neuropsychol. 22, 262-275. doi: 10.1080/02643290442000095

Raichle, M. E. (2015). The brain's default mode network. Annu. Rev. Neurosci. 38, 433-447. doi: 10.1146/annurev-neuro-071013-014030 
Raichle, M. E., Macleod, A. M., Snyder, A. Z., Powers, W. J., Gusnard, D. A., and Shulman, G. L. (2001). A default mode of brain function. Proc. Natl. Acad. Sci. U.S.A. 98, 676-682. doi: 10.1073/pnas.98.2.676

Reilly, J., Peelle, J. E., Garcia, A., and Crutch, S. J. (2016). Linking somatic and symbolic representation in semantic memory: the dynamic multilevel reactivation framework. Psychon. Bull. Rev. 23, 1002-1014. doi: 10.3758/s13423-015-0824-5

Renoult, L., Davidson, P. S., Palombo, D. J., Moscovitch, M., and Levine, B. (2012). Personal semantics: at the crossroads of semantic and episodic memory. Trends Cogn. Sci. 16, 550-558. doi: 10.1016/j.tics.2012.09.003

Rice, G. E., Lambon Ralph, M. A., and Hoffman, P. (2015). The roles of left versus right anterior temporal lobes in conceptual knowledge: an ALE metaanalysis of 97 functional neuroimaging studies. Cereb. Cortex 25, 4374-4391. doi: 10.1093/cercor/bhv024

Robson, H., Sage, K., and Ralph, M. A. L. (2012). Wernicke's aphasia reflects a combination of acoustic-phonological and semantic control deficits: a case-series comparison of Wernicke's aphasia, semantic dementia and semantic aphasia. Neuropsychologia 50, 266-275. doi: 10.1016/j.neuropsychologia.2011.11.021

Rogers, T. T., Lambon Ralph, M. A., Garrard, P., Bozeat, S., McClelland, J. L., Hodges, J. R., et al. (2004). Structure and deterioration of semantic memory: a neuropsychological and computational investigation. Psychol. Rev. 111, 205-235. doi: 10.1037/0033-295X.111.1.205

Sabsevitz, D. S., Medler, D. A., Seidenberg, M., and Binder, J. R. (2005). Modulation of the semantic system by word imageability. Neuroimage 27, 188-200. doi: 10.1016/j.neuroimage.2005.04.012

Salvador, R., Suckling, J., Coleman, M. R., Pickard, J. D., Menon, D., and Bullmore, E. (2005). Neurophysiological architecture of functional magnetic resonance images of human brain. Cereb. Cortex 15, 1332-1342. doi: 10.1093/cercor/bhi016

Saur, D., Kreher, B. W., Schnell, S., Kümmerer, D., Kellmeyer, P., Vry, M.-S., et al. (2008). Ventral and dorsal pathways for language. Proc. Natl. Acad. Sci. U.S.A. 105, 18035-18040. doi: 10.1073/pnas.0805234105

Schacter, D. L., Addis, D. R., and Buckner, R. L. (2007). Remembering the past to imagine the future: the prospective brain. Nat. Rev. Neurosci. 8, 657-661. doi: $10.1038 /$ nrn2213

Schwartz, M. F., Kimberg, D. Y., Walker, G. M., Brecher, A., Faseyitan, O. K., Dell, G. S., et al. (2011). Neuroanatomical dissociation for taxonomic and thematic knowledge in the human brain. Proc. Natl. Acad. Sci. U.S.A. 108, 8520-8524. doi: $10.1073 /$ pnas.1014935108

Seghier, M. L. (2013). The angular gyrus multiple functions and multiple subdivisions. Neuroscientist 19, 43-61. doi: 10.1177/1073858412440596

Seghier, M. L., Fagan, E., and Price, C. J. (2010). Functional subdivisions in the left angular gyrus where the semantic system meets and diverges from the default network. J. Neurosci. 30, 16809-16817. doi: 10.1523/JNEUROSCI.3377-10.2010

Sepulcre, J., Sabuncu, M. R., Yeo, T. B., Liu, H., and Johnson, K. A. (2012). Stepwise connectivity of the modal cortex reveals the multimodal organization of the human brain. J. Neurosci. 32, 10649-10661. doi: 10.1523/JNEUROSCI.0759-12.2012

Singh, K. D., and Fawcett, I. P. (2008). Transient and linearly graded deactivation of the human default-mode network by a visual detection task. Neuroimage 41, 100-112. doi: 10.1016/j.neuroimage.2008.01.051

Sporns, O. (2013). Network attributes for segregation and integration in the human brain. Curr. Opin. Neurobiol. 23, 162-171. doi: 10.1016/j.conb.2012.11.015

Sporns, O., and Betzel, R. F. (2016). Modular brain networks. Annu. Rev. Psychol. 67:613. doi: 10.1146/annurev-psych-122414-033634

Spreng, R. N., Mar, R. A., and Kim, A. S. (2009). The common neural basis of autobiographical memory, prospection, navigation, theory of mind, and the default mode: a quantitative meta-analysis. J. Cogn. Neurosci. 21, 489-510. doi: 10.1162/jocn.2008.21029

Sugiura, M., Sassa, Y., Watanabe, J., Akitsuki, Y., Maeda, Y., Matsue, Y., et al. (2006). Cortical mechanisms of person representation: recognition of famous and personally familiar names. Neuroimage 31, 853-860. doi: 10.1016/j.neuroimage.2006.01.002

Thompson, H. E., Robson, H., Ralph, M. L., and Jefferies, E. (2015). Varieties of semantic 'access' deficit in Wernicke's aphasia and semantic aphasia. Brain 138, 3776-3792. doi: 10.1093/brain/awv281

Turken, U., and Dronkers, N. F. (2011). The neural architecture of the language comprehension network: converging evidence from lesion and connectivity analyses. Front. Syst. Neurosci. 5:1. doi: 10.3389/fnsys.2011.00001 van den Heuvel, M. P., and Sporns, O. (2013). Network hubs in the human brain. Trends Cogn. Sci. 17, 683-696. doi: 10.1016/j.tics.2013.09.012

Vandenberghe, R., Wang, Y., Nelissen, N., Vandenbulcke, M., Dhollander, T., Sunaert, S., et al. (2013). The associative-semantic network for words and pictures: effective connectivity and graph analysis. Brain Lang. 127, 264-272. doi: 10.1016/j.bandl.2012.09.005

Vigliocco, G., and Vinson, D. P. (2007). "Semantic representation," in The Oxford Handbook of Psycholinguistics, ed M. G. Gaskell (Oxford: Oxford University Press), 195-215. doi: 10.1093/oxfordhb/9780198568971.013.0012

Vigliocco, G., Meteyard, L., Andrews, M., and Kousta, S. (2009). Toward a theory of semantic representation. Lang. Cogn. 1, 219-247. doi: 10.1515/LANGCOG.2009.011

Vincent, J. L., Kahn, I., Snyder, A. Z., Raichle, M. E., and Buckner, R. L. (2008). Evidence for a frontoparietal control system revealed by intrinsic functional connectivity. J. Neurophysiol. 100, 3328-3342. doi: 10.1152/jn.903 55.2008

Visser, M., Jefferies, E., and Ralph, M. L. (2010). Semantic processing in the anterior temporal lobes: a meta-analysis of the functional neuroimaging literature. $J$. Cogn. Neurosci. 22, 1083-1094. doi: 10.1162/jocn.2009.21309

Wang, J., Conder, J. A., Blitzer, D. N., and Shinkareva, S.V. (2010). Neural representation of abstract and concrete concepts: a metaanalysis of neuroimaging studies. Hum. Brain Mapp. 31, 1459-1468. doi: 10.1002/hbm.20950

Wang, X., Peelen, M. V., Han, Z., Caramazza, A., and Bi, Y. (2016). The role of vision in the neural representation of unique entities. Neuropsychologia 87, 144-156. doi: 10.1016/j.neuropsychologia.2016.05.007

Warren, D. E., Power, J. D., Bruss, J., Denburg, N. L., Waldron, E. J., Sun, H., et al. (2014). Network measures predict neuropsychological outcome after brain injury. Proc. Natl. Acad. Sci. U.S.A. 111:14247. doi: 10.1073/pnas.1322173111

Wei, T., Liang, X., He, Y., Zang, Y., Han, Z., Caramazza, A., et al. (2012). Predicting conceptual processing capacity from spontaneous neuronal activity of the left middle temporal gyrus. J. Neurosci. 32, 481-489. doi: 10.1523/JNEUROSCI.1953-11.2012

Whitney, C., Kirk, M., O'sullivan, J., Lambon Ralph, M. A., and Jefferies, E. (2011). The neural organization of semantic control: TMS evidence for a distributed network in left inferior frontal and posterior middle temporal gyrus. Cereb. Cortex 21, 1066-1075. doi: 10.1093/cercor/bhq180

Whitney, C., Kirk, M., O'sullivan, J., Ralph, M. L., and Jefferies, E. (2012). Executive semantic processing is underpinned by a large-scale neural network: revealing the contribution of left prefrontal, posterior temporal, and parietal cortex to controlled retrieval and selection using TMS. J. Cogn. Neurosci. 24, 133-147. doi: 10.1162/jocn_a_00123

Wirth, M., Jann, K., Dierks, T., Federspiel, A., Wiest, R., and Horn, H. (2011). Semantic memory involvement in the default mode network: a functional neuroimaging study using independent component analysis. Neuroimage 54, 3057-3066. doi: 10.1016/j.neuroimage.2010.10.039

Xu, Y., Lin, Q., Han, Z., He, Y., and Bi, Y. (2016). Intrinsic functional network architecture of human semantic processing: modules and hubs. Neuroimage 132, 542-555. doi: 10.1016/j.neuroimage.2016.03.004

Yee, E., Chrysikou, E. G., and Thompson-Schill, S. L. (2013). "Semantic memory," in The Oxford Handbook of Cognitive Neuroscience, Vol. 1, Core Topics, eds K. N. Ochsner and S. Kosslyn (Oxford: Oxford University Press), 353-374. doi: 10.1093/oxfordhb/9780199988693.013.0017

Yeo, B. T., Krienen, F. M., Sepulcre, J., Sabuncu, M. R., Lashkari, D., Hollinshead, M., et al. (2011). The organization of the human cerebral cortex estimated by intrinsic functional connectivity. J. Neurophysiol. 106, 1125-1165. doi: 10.1152/jn.00338.2011

Zwaan, R. A. (2014). Embodiment and language comprehension: reframing the discussion. Trends Cogn. Sci. 18, 229-234. doi: 10.1016/j.tics.2014.02.008

Conflict of Interest Statement: The authors declare that the research was conducted in the absence of any commercial or financial relationships that could be construed as a potential conflict of interest.

Copyright (C) $2017 \mathrm{Xu}$, He and Bi. This is an open-access article distributed under the terms of the Creative Commons Attribution License (CC BY). The use, distribution or reproduction in other forums is permitted, provided the original author(s) or licensor are credited and that the original publication in this journal is cited, in accordance with accepted academic practice. No use, distribution or reproduction is permitted which does not comply with these terms. 